\title{
Responsabilidad Social Corporativa y Rendimiento Financiero: un Meta-Análisis
}

\section{María del Mar Miras Rodríguez, Amalia Carrasco Gallego \& Bernabé Escobar Pérez}

To cite this article: María del Mar Miras Rodríguez, Amalia Carrasco Gallego \& Bernabé Escobar Pérez (2014) Responsabilidad Social Corporativa y Rendimiento Financiero: un Meta-Análisis, Spanish Journal of Finance and Accounting / Revista Espanola de Financiacion y Contabilidad, 43:2, 193-215, DOI: $10.1080 / 02102412.2014 .911000$

To link to this article: https://doi.org/10.1080/02102412.2014.911000

\section{册 Published online: 20 May 2014.}

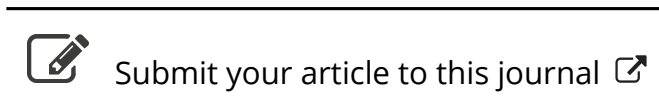

Џlll Article views: 633

View Crossmark data ¿

Citing articles: 4 View citing articles $\square$ 


\title{
Responsabilidad Social Corporativa y Rendimiento Financiero: un Meta-Análisis
}

\section{Corporate social responsibility and financial performance: a meta-analysis}

\author{
María del Mar Miras Rodríguez*, Amalia Carrasco Gallego and Bernabé Escobar Pérez \\ Departamento de Contabilidad y Economía Financiera. Universidad de Sevilla
}

(Received 23 December 2011; accepted 13 February 2014)

A pesar de que el concepto de Responsabilidad Social no ha variado sustancialmente desde que fuese definido por Carroll (1979), sí que tanto la implantación de las prácticas socialmente responsables y su papel en la estrategia de las empresas, como la manera en la que las empresas informan sobre ellas ha cambiado notablemente durante lo que llevamos de siglo XXI. Esto unido al gran esfuerzo que están realizando las empresas por ser socialmente responsables hace que sea más interesante que nunca el estudio de la relación entre RSC y Rendimiento Financiero (RF) y, en concreto, el estudio del posible efecto moderador que determinadas variables como el tipo de acciones de RSC que se estudian (según el enfoque Triple Bottom Line), la forma de reporting utilizada, la medida de $\mathrm{RF}$ y el ámbito espacial; puedan ejercer sobre ella. Basándonos en una muestra de 86 estimaciones procedentes de artículos publicados a partir del año 2000, vamos a tratar de ver si existe relación entre la Responsabilidad Social y el Rendimiento Financiero, así como estudiar si las variables moderadoras planteadas nos permiten avanzar en su conocimiento.

Palabras clave: Responsabilidad Social Corporativa; Rendimiento Financiero; Metaanálisis

Though the concept of corporate social responsibility (CSR) has not changed substantially since it was defined by Carroll (1979) since the beginning of the century, there have been considerable changes in the implementation of CSR practices and their role in corporate strategy as well as in the way in which they have been reported. This, together with the great effort that companies are making to be socially responsible, makes the study of the relationship between CSR and financial performance and that of the possible moderating effect which some variables - the type of CSR activities (according to the Triple Bottom Line), the CSR Reporting, the RF measurement and the spatial scope - may have on it even more interesting. Based on a sample of 86 estimations from articles published since 2000 , we will try to see whether there is a relationship between these two variables, and if the proposed moderator variables allow us to achieve a greater knowledge of this.

Keywords: corporate social responsibility; financial performance; meta-analysis 


\section{Introducción}

La Responsabilidad Social Corporativa (en adelante RSC) ha irrumpido con fuerza en las empresas pasando de ser un objetivo bonito e idealista del que se hablaba en la literatura académica a una realidad y a uno de los objetivos que tiene más peso en la toma de decisiones (De la Cuesta, 2004; Garriga \& Melé, 2004; Nieto \& Fernández, 2004).

Prueba de ello son las diferentes iniciativas que corroboran su relevancia creciente en el siglo XXI: la regulación del concepto de RSC por parte de organismos internacionales (Libro Verde de Responsabilidad Social de la Unión Europea en 2001 y las distintas iniciativas de RSC llevadas a cabo por la Comisión Europea a partir de 2002), el aumento de las empresas que publican memorias de sostenibilidad con carácter voluntario y la creación de índices bursátiles dependientes de la RSC (Dow Jones Sustainability Index DJSI-, KLD Domini Social Index y FTSE4Good Index).

Todo ello nos hace plantearnos su importancia y las razones por las que se está implantando en las empresas, las cuales probablemente vayan más allá del mero altruismo y la búsqueda del bien común, necesitando una justificación económica para poder llevarse a cabo. En esta línea, Burke and Logsdon (1996), Husted and Allen (2000) y McWilliams and Siegel (2001), entre otros, llegan a abogar por la RSC como inversión estratégica de las empresas, ya que puede llevarles a desarrollar capacidades únicas que les permitan desarrollar una ventaja competitiva sostenible (Toro, 2006).

Precisamente, a pesar de que esta relación entre la RSC y Rendimiento Financiero (en adelante RF) ha sido ampliamente estudiada en la literatura -revisiones bibliográficas (Beurden \& Gössling, 2008; Fifka, 2013) y meta-análisis (Orlitzky, Schmidt, \& Rynes, 2003; Allouche \& Laroche, 2005; Wu, 2006)-, los resultados alcanzados son heterogéneos y no nos permiten explicar de forma satisfactoria dicha relación.

Estos trabajos agrupan la evidencia encontrada hasta el momento en el que se realizan, considerando la RSC como un concepto estático que no varía con el paso del tiempo. Sin embargo, aunque lo que se entiende por RSC no ha variado sustancialmente desde los años 70, soliéndose utilizar la definición de Carroll (1979), sí que tanto la implantación de las prácticas de RSC en las empresas, como la manera en la que se informa sobre ellas, ha cambiado ostensiblemente, pasando a ser obligatoria en algunos países. Por ello, resulta más adecuado estudiar un periodo concreto, en el cual la implementación de las prácticas y el reporting no hayan variado de forma significativa.

Adicionalmente, durante el siglo XXI han proliferado los trabajos que tratan de encontrar los factores que influyen en esta relación, ampliando además el ámbito espacial de estudio, el cual se reducía hasta comienzos del siglo XXI a EE.UU y Reino Unido.

Por ello, vamos a analizar de nuevo la relación entre las prácticas de RSC y el RF, mediante un meta-análisis que introduce diversas novedades frente a los trabajos precedentes (Orlitzky et al., 2003; Allouche \& Laroche, 2005; Wu, 2006), en cuanto a la muestra utilizada y a las variables moderadoras que se estudian.

Respecto a la muestra, vamos a estudiar la relación en el horizonte temporal del siglo XXI. Este hecho se debe, en primer lugar a la mayor homogeneidad en la implantación de las prácticas de RSC. También resulta relevante para la elección del horizonte temporal, las numerosas iniciativas de reporting de RSC que surgen a principio de este siglo, las cuales cambian radicalmente la forma de valorar la RSC y proponen un marco regulatorio de la misma aunque no sea de obligado cumplimiento para las empresas (índices bursátiles, GRI,..). Adicionalmente, la elección de dicho periodo temporal permite la inclusión de estudios procedentes de 28 países, lo que nos proporciona una evidencia más global que nos posibilita realizar comparaciones entre países. 
Por otro lado, debido a que numerosos autores (Van der Laan, Van Ees, \& Van, 2008; Prior, Surroca, \& Tribó., 2008, entre otros) argumentan la conveniencia de la introducción de variables moderadoras para ayudar a clarificar la relación RSC-RF, estudiaremos las siguientes: en primer lugar, diferenciamos entre aquellos trabajos que valoran dimensiones específicas del Enfoque de la Triple Bottom Line, frente a aquellos que la valoran de forma global, además de tener en cuenta la forma en la que se valora dicha información para relacionarla con el RF. En segundo lugar, tenemos en cuenta la forma escogida para medir el RF en dichos trabajos. Por último, partiendo de la diversidad de países con la que contamos en la muestra y debido a la influencia que la cultura nacional podría tener sobre la RSC (Waldman, de Luque, Washburn, \& House, 2006), agrupamos los trabajos en función de diversas clasificaciones culturales.

Por todo ello, el objetivo de nuestro trabajo es aportar evidencia sobre la existencia de una relación positiva entre las acciones de RSC y el RF, así como sobre el efecto que las variables moderadoras mencionadas puedan ejercer sobre ella.

Así, la estructura de nuestro trabajo queda configurada de la siguiente manera. En el apartado 2 nos centramos en el debate sobre la relación entre RSC y RF y, en consecuencia, formularemos las hipótesis a contrastar. El apartado 3 está dedicado a la explicación de la metodología empleada, la selección de la muestra y las variables utilizadas. Por su parte, en el apartado 4, comentaremos los resultados y los analizaremos, para finalmente, en el apartado 5 exponer las conclusiones, las limitaciones del estudio, así como algunas futuras líneas de investigación.

\section{Responsabilidad Social Corporativa y Rendimiento Financiero}

El marco teórico en el que se fundamenta la relación entre RSC y RF está formado por numerosas teorías que tratan de explicar todas las posibles relaciones causales, así como su carácter positivo o negativo (Preston \& O’Bannon, 1997). Así, mientras la influencia que el RF obtenido por las empresas pudiera tener sobre sus comportamientos socialmente responsables vendría explicada por la Hipótesis de la Escasez de Recursos (Waddock \& Graves, 1997) y la Hipótesis del Oportunismo de los Directivos (Williamson, 1965), los posibles efectos de las acciones de RSC realizadas sobre el RF se fundamentarían en la Hipótesis del Impacto Social (Freeman, 1984) y la Hipótesis del Trade-Off (Friedman, 1970). Adicionalmente, numerosos investigadores están considerando que existen sinergias entre ambas variables (positivas o negativas) y que, por tanto, la relación tiene un carácter bidireccional.

A pesar de ser un tema que ha sido ampliamente abordado en la literatura empírica, los resultados encontrados son de naturaleza muy diversa y no permiten concluir claramente sobre su existencia (Ruf, Muralidhar, Brown, Janney, \& Paul, 2001), ni sobre el sentido de la misma (Salzmann, Ionescu-Somers, \& Steger, 2005).

La heterogeneidad encontrada en los resultados de los diferentes trabajos que abordan esta temática, ha hecho que sea extensa la literatura que trata de aclarar su existencia y carácter (influencia positiva o negativa), así como su sentido (cuál de las dos variables es la dependiente), tanto a través de la compilación de los resultados -revisiones bibliográficas (Brammer \& Millington, 2005; Fernández \& Luna, 2007; Beurden \& Gössling, 2008) y meta-análisis (Orlitzky et al., 2003; Allouche \& Laroche, 2005; Wu, 2006)-, como introduciendo ciertos factores moderadores o mediadores en el análisis que pueden ayudar a comprender mejor qué ocurre realmente en esa relación (Gómez, 2008). 
Tabla 1. Comparativa entre los meta-análisis revisados.

\begin{tabular}{lccl}
\hline & \multicolumn{3}{c}{ CARACTERÍSTICAS } \\
\cline { 2 - 4 } META-ANÁLISIS & Número de estudios & Horizonte Temporal & Ámbito Espacial \\
\hline REVISADOS & 52 & $1972-1997$ & EE.UU. \\
Orlitzky et al. (2003) & 82 & $1972-2003$ & EE.UU. \\
Allouche \& Laroche (2005) & $121(39)$ & $1975-1999$ & Reino Unido \\
Wu (2006) & & & EE.UU. \\
\hline
\end{tabular}

Fuente: Elaboración propia.

Con el objetivo de extraer una conclusión que fuese más homogénea sobre la relación, Orlitzky et al. (2003), Allouche and Laroche (2005) y Wu (2006) realizaron sendos metaanálisis, cuyas características más significativas podemos observar de forma comparativa en la Tabla 1.

Basándose principalmente en la Hipótesis del Impacto Social (Freeman, 1984), en la Hipótesis de Escasez de Recursos (Waddock \& Graves, 1997) y en Teoría de la Agencia (Ross, 1973; Eisenhardt, 1989), dichos artículos llegan a concluir que existe una relación positiva entre las variables RSC y RF. Sin embargo, reconocen expresamente que a pesar del avance en el conocimiento de la relación, queda patente que es necesario profundizar sobre su naturaleza, ya que queda mucho por explicar.

Consecuentemente, la primera hipótesis de nuestro trabajo es confirmar dichos resultados positivos:

$H_{1}$ : Existe una relación positiva entre las políticas de RSC y el RF de las empresas.

Para profundizar en esta relación, son muchos los trabajos que plantean la conveniencia de introducir ciertas variables moderadoras o mediadoras de esta relación que permitirían conocerla mejor (Van der Laan et al., 2008; Prior et al., 2008; Hull \& Rothenberg, 2008; Dunn \& Sainty, 2009; Surroca, Tribó, \& Waddock, 2010).

Por tanto, el objeto de nuestro estudio será identificar algunos de los factores que afectan a la relación entre RSC y RF, tomando como punto de partida los identificados por Orlitzky et al. (2003), Allouche and Laroche (2005) y Wu (2006), todos ellos referidos a la forma de medir ambas variables.

Por ello, vamos a estudiar el efecto que tanto (1) el tipo de acciones de RSC que se estudian (Triple Bottom Line), (2) la forma de Reporting utilizada por la empresa o por el investigador, (3) la medida de RF utilizada, (4) si se tienen en cuenta los retardos temporales o no, y (5) el ámbito espacial, ejercen sobre la relación.

\subsection{Enfoque Triple Bottom Line}

El enfoque Triple Bottom Line (Elkington, 1998) se basa en la Teoría de los Stakeholders, al asumir que las responsabilidades de la empresa abarcan aspectos que van mucho más allá de los estrictamente económicos, como son los sociales y los medioambientales (Hubbard, 2009). 
De este modo, este enfoque permite que acotemos y diferenciemos dentro del concepto genérico de RSC, los aspectos sociales y los medioambientales. Por un lado, las acciones sociales se refieren al impacto que una empresa ejerce sobre las comunidades en las que trabaja, mientras que las políticas medioambientales están orientadas hacia la cantidad de recursos utilizados y a los residuos generados por su actividad.

En la literatura especializada, se pone de manifiesto que el comportamiento de las acciones sociales y medioambientales de las empresas respecto al RF es muy diferente por un doble motivo. En primer lugar, aunque el concepto de voluntariedad es fundamental para la consideración de una acción socialmente responsable (Carroll, 1991; Dahlsrud, 2008), encontramos un marco normativo medioambiental muy desarrollado en la mayoría de los países, mientras que las acciones sociales prácticamente no están reguladas, siendo dicho tema objeto de numerosos debates en la literatura (Williamson, Lynch-Wood, \& Ramsay, 2006). La existencia de las exigentes regulaciones medioambientales obliga a las empresas a ser proactivas, debido a los altos costes que un cambio normativo lleva aparejados si no ha sido anticipado por la empresa.

En segundo lugar, según la Teoría de la Eco-Eficiencia (Porter \& Van der Linde, 1995) la implantación de nuevas máquinas y/o instalaciones supone unos ahorros de costes energéticos y de recursos, o menor gasto por la eliminación de residuos para las empresas. En cambio, los beneficios empresariales derivados de las políticas sociales (comunidad, empleados, proveedores) no son tan directos e inmediatos para la empresa, ya que éstos vienen por la coincidencia entre las expectativas de los grupos de interés y lo que las empresas les ofrecen según, la Hipótesis del Impacto Social (Freeman, 1984).

Por otro lado, la filantropía o las donaciones han sido consideradas como una parte importante de las acciones sociales orientadas a la comunidad. Sin embargo, su comportamiento se revela completamente diferente al del resto de acciones sociales, ya que se trata de un coste cuya recuperación no es tan rápida como en el resto de acciones sociales y/o medioambientales, ya que no supone un cambio interno en la forma de hacer las cosas en las compañías. El retorno de las donaciones viene a través del impacto positivo que éstas tienen sobre la reputación de la compañía (Brammer \& Millington, 2005).

Por tanto, vamos a contrastar si considerar aspectos concretos de la RSC (Social, Medioambiental, Filantropía) en lugar de estudiarla en su conjunto afecta a su relación con el RF:

$H_{2}$ : Los aspectos de la RSC considerados en los estudios (Social, Medioambiental, Filantrópico) moderan la relación entre RSC y $R F$.

\section{2. Ámbito espacial}

Numerosos investigadores han puesto de manifiesto la influencia significativa que la cultura nacional ejerce sobre las acciones de RSC que llevan a cabo las empresas (Ringov \& Zollo, 2007), sobre la revelación de información sobre RSC (Waldman et al., 2006; Prado y García, 2011), y/o sobre el comportamiento ético de las compañías (Fernández \& Luna, 2007; Singh, Salmones Sanchez, \& Del Bosque, 2008; Yong, 2008; Svensson, Wood, Singh, Carasco, \& Callaghan, 2009).

Dichos trabajos se basan en los argumentos dados por la Teoría Institucional (Baughn, Body, \& McIntosh, 2007; Matten \& Moon, 2008), la cual se centra en comparar las motivaciones que tienen los directivos a realizar acciones de RSC 
dependiendo del contexto cultural en el que se encuentren (Aguilera, Rupp, Williams, \& Ganapathi, 2007).

Esto implica directamente que existen diferencias relativas a cómo la RSC es entendida en cada país (Jamali \& Mirshak, 2007), las cuales afectan, por tanto, a la importancia que dichas acciones tengan para la estrategia de sus empresas. Así pues, la relación entre la RSC y el RF puede verse afectada por el ámbito espacial donde éstas se desarrollan (Gray, Javad, Power, \& Sinclair, 2001; Scholtens \& Kang, 2013).

Como consecuencia, formulamos la tercera hipótesis como sigue:

$H_{3}$ : El país al que pertenecen las empresas analizadas tiene un efecto moderador sobre la relación $R S C-R F$

\section{Metodología}

El meta-análisis es una técnica estadística que permite integrar cuantitativamente los resultados de los estudios previos sobre un tema de investigación en concreto, para poder obtener una conclusión generalizable al respecto (Sánchez-Meca, 2008; GarcíaMeca \& Sánchez-Ballesta, 2010). Surge con los trabajos de Schmidt and Hunter (1977) y Smith and Glass (1977) en el campo de la Psicología. Posteriormente ha sido utilizado tanto en estudios de ámbito contable (García-Meca and Sánchez-Ballesta, 2006) como en estudios referidos a la relación entre RSC y RF (Orlitzky et al., 2003; Allouche \& Laroche, 2005; Wu, 2006), ya que es de especial utilidad en campos donde los resultados encontrados hasta el momento son muy heterogéneos y no permiten alcanzar conclusiones sólidas sobre las evidencias científicas obtenidas en las investigaciones previas (Rosenthal, 1991).

Según Sánchez-Meca (2010) esta técnica introduce significativas ventajas frente a la tradicional revisión bibliográfica, que denomina revisiones subjetivas, y sobre otras revisiones sistemáticas (por ejemplo, el vote-counting review), siendo la más importante la introducción de una valoración cuantitativa y estadística de los resultados a través del denominado "tamaño efecto" definido por Cohen (1969, p.23) como "el grado en el que el fenómeno objeto de estudio está presente en una población”.

Así, tras plantear claramente el problema de investigación que queremos investigar, los siguientes pasos serían la búsqueda bibliográfica de los estudios a incluir y su correspondiente codificación, el cálculo del tamaño efecto (teniendo en cuenta la elección del estadístico para medir el tamaño efecto), la evaluación de la homogeneidad de los resultados y, por último, indagar si dicha variabilidad se debe al efecto moderador que determinadas variables ejercen sobre la relación que se está estudiando.

\subsection{Revisión bibliográfica}

Para seleccionar los trabajos a analizar hemos utilizado diversas fuentes de información teniendo en cuenta el horizonte temporal que vamos a estudiar (2000-2012). En primer lugar, hemos realizado una búsqueda en la base de datos ISI Web of Knowledge, por contener los artículos de las revistas incluidas en el JCR (Journal Citation Report), así como algunas aportaciones a congresos, a través de los términos "Corporate Social Responsibility, Financial Performance, Analysis" y "Corporate Social Responsibility, Financial Performance, Empirical", con el objetivo de eliminar parte de los artículos de corte teórico. Para complementar dicha fuente, hemos incluido artículos de las revisiones literarias realizadas por Beurden and Gössling (2008) y Fifka (2013). Por último, para 
superar el sesgo de publicación ${ }^{1}$ (Kirkham \& Dwan, 2010) se han incluido en la muestra estudios procedentes de $\mathrm{SSRN}^{2}$.

Una vez recopilados los trabajos, eliminamos las posibles duplicidades que podían existir por haber empleado distintas fuentes. Posteriormente, fueron excluidas diversas aportaciones debido a su carácter teórico y otras que no informaban acerca del estadístico elegido (coeficiente de correlación de Pearson) o bien no se podían transformar en éste a través de las fórmulas propuestas por Wolf (1986), Rosenthal (1991) y Lipsey and Wilson (2001).

Con este proceso hemos obtenido 86 muestras independientes procedentes de 70 artículos que estudian la relación entre la RSC y el RF (Anexo 1). Esta diferencia entre el número de muestras y artículos se debe a que tanto Inoue and Lee (2011) como Usunier, Furrer, and Furrer-Perrinjaquet (2011) utilizan en sus trabajos muestras independientes, ya que estudian dicha relación en dos sectores distintos y en dieciséis países diferentes respectivamente.

\subsection{Tamaño efecto y método}

Para llevar a cabo el análisis hemos escogido entre los distintos enfoques meta-analíticos, la técnica desarrollada por Hunter y Schmidt (1990), por ser la más utilizada en Economía, así como en otros trabajos meta-analíticos sobre RSC (Orlitzky et al., 2003; Allouche \& Laroche, 2005; Wu, 2006). Por tanto, el estadístico utilizado para medir el tamaño efecto es el coeficiente de correlación de Pearson (r).

Con el objeto de estimar el tamaño efecto medio, hemos obtenido un coeficiente de correlación de Pearson por cada muestra independiente incluida en el estudio. Esto implica localizar los coeficientes de correlación de Pearson o sus transformaciones pertinentes (a través de las fórmulas de Wolf, 1986; Rosenthal, 1991; Lipsey \& Wilson, 2001; $r=\left[\mathrm{t}^{2} /\left(\mathrm{t}^{2}\right.\right.$ $\left.+\mathrm{gl})]^{1 / 2}, \mathrm{r}=[\mathrm{F} /(\mathrm{F}+\mathrm{gl})]^{1 / 2}\right)$ en los estudios y obtener un coeficiente ponderado por cada uno de ellos para garantizar la independencia de las muestras consideradas (Lipsey \& Wilson, 2001), ya que la gran mayoría de los trabajos ofrecen varios coeficientes dependiendo de la forma de medir el RF o la RSC. En los análisis por subgrupos en los que incluyan estas medidas diferentes, usaremos los coeficientes originales aunque manteniendo una correlación por estudio y subgrupo.

Una vez calculado el tamaño de efecto medio $\left(\mathrm{r}^{*}=\sum\left(\mathrm{n}_{\mathrm{i}} * \mathrm{r}_{\mathrm{i}}\right) / \sum \mathrm{n}_{\mathrm{i}}\right)$, hemos estimado sus correspondientes medidas de bondad de ajuste a través de la definición de un intervalo de confianza del $95 \%$ y realizado un doble examen de la homogeneidad de los resultados: (1) "la regla del $75 \%$ ”, según la cual si el $75 \%$ de la varianza observada $\left(\mathrm{S}_{\mathrm{r}}^{2}=\sum \mathrm{n}_{\mathrm{i}}\left(\mathrm{r}_{\mathrm{i}}-\mathrm{r}^{*}\right)^{2} / \sum \mathrm{n}_{\mathrm{i}}\right)$ se explica por el error de muestreo $\left(\mathrm{S}_{\mathrm{e}}^{2}=\right.$ $\left.\left(1-r^{* 2}\right)^{2} \mathrm{k} / \sum \mathrm{n}_{\mathrm{i}}\right)$, se asume que los resultados son homogéneos; y (2) el estadístico Q de homogeneidad de (Hedge \& Olkin, 1985; $\left.\mathrm{Q}=\sum \mathrm{n}_{\mathrm{i}}\left(\mathrm{r}-\mathrm{r}^{*}\right)^{2}\right)$ que sigue una distribución chi-cuadrado con n-1 grados de libertad, cuya significatividad supone el rechazo de la hipótesis nula, es decir, que los estudios son heterogéneos. Así mismo, se han incluido las pruebas de diferencias de medias (F de Ficher-Snedecord para medias y varianzas conocidas) dentro de los grupos.

\subsection{Variables y efectos moderadores}

Como ya se ha señalado, en la literatura sobre la relación entre la RSC y el RF, se constata que la introducción de determinadas variables como moderadoras de la relación en el 
análisis pueden reducir la heterogeneidad $\mathrm{y}$, por tanto, ayudar a alcanzar mejores conclusiones.

En este sentido, tal y como se comentó en el apartado 2, podemos distinguir entre: (1) las diferencias en la forma de valorar la RSC, (2) las distintas maneras de medir el RF, (3) los momentos temporales del análisis y, por último, (4) los países de estudio en cada uno de las muestras.

Refiriéndonos a la forma de valorar la RSC, realizamos una doble clasificación. Por un lado, siguiendo las razones argumentadas por el enfoque del desarrollo sostenible o de la Triple Bottom Line, diferenciamos entre los trabajos que se centran en aspectos sociales, medioambientales, económicos, o bien que abarcan todas las dimensiones anteriores no haciendo diferencia alguna. Por otro lado, encontramos diversas formas de valorar la información de RSC proporcionada por las empresas y, por ello, vamos a diferenciar entre los trabajos dependiendo de si la valoración de la RSC se basa en el disclosure (es decir, si la empresa informa sobre si realiza o no determinadas prácticas), en las evaluaciones del compromiso de las empresas con las diferentes prácticas (habitualmente a través de encuestas vía escalas de Likert), en las cantidades monetarias que las empresas destinan a RSC y, por último, en función de su pertenencia a índices bursátiles ligados al comportamiento socialmente responsable (DJSI, FTSE4GOOD, entre otros).

En primer lugar, para elaborar los índices basados en disclosure se realiza un análisis de contenido de la información proporcionada por las propias empresas, en las memorias de sostenibilidad $u$ otros documentos elaborados, en el cual se asigna el valor 1 si informa sobre las prácticas y 0 si no lo hace (Hackston \& Milne, 1996; Hughes, Anderson, \& Golden, 2001; Al-Tuwaijri, Chistensen, \& Hughes, 2004), o bien se valora la calidad de ésta información de 0 a 4, en función de su adecuación al GRI (Prado-Lorenzo, GallegoAlvarez, \& García-Sánchez, 2009).

En segundo lugar, nos encontramos con la valoración de la información que las empresas dan como respuesta a diversos cuestionarios. Dicha valoración, habitualmente una escala de Likert, obliga a la empresa a informar sobre todos los aspectos que se le preguntan y da una visión que admite diferentes niveles en los que una acción concreta pueda estar implantada en la compañía. Por otro lado, no deja de ser información autorevelada, con el consiguiente sesgo a favor de la compañía que puede suponer.

Basándose en la evaluación de la información auto-revelada, junto a la que se encuentra en las memorias y documentos, las empresas pasan a formar parte de los índices de sostenibilidad o no. Éstos índices son dinámicos, ya que las empresas son evaluadas periódicamente, y dependiendo del resultado de dicha evaluación entran o salen de los índices (Cho, Guidry, Hageman, \& Patten, 2012). En la literatura es una forma bastante utilizada para distinguir entre las empresas que se encuentran en dichos índices sostenibles y las que no. Esta forma de valorar la RSC de las empresas ha sido objeto de numerosas críticas en la literatura, por la elevada confianza que se deposita en la información proporcionada por la empresa (Fowler \& Hope, 2007), es decir, porque dan más importancia a lo que las empresas dicen que a lo que realmente hacen en materia de RSC (Crane \& Matten, 2007).

Por último, nos encontramos con algunos trabajos (Lima, de Souza, \& Cortes de Vasconcellos, 2011; Cabeza-García, Martínez-Campillo, \& Marbella-Sánchez, 2010) que valoran la información socialmente responsable por las salidas directas de tesorería que suponen, no pudiéndose encuadrar en ninguno de los grupos anteriores. Así pues, es de esperar que la relación entre RSC y RF se vea afectada por la forma en la que los investigadores valoran las prácticas de RSC de las empresas. 
En cuanto al RF, son numerosas las alternativas que se utilizan para medir el RF en la literatura en general, y, en particular, en los trabajos que miden su relación con la RSC. Este hecho ha sido estudiado anteriormente (Orlitzky et al., 2003; Allouche \& Laroche, 2005; Wu, 2006, entre otros), evidenciando que no existe consenso en la forma de medir el RF que afecta o se ve afectada por las acciones de RSC.

En los trabajos incluidos en la muestra podemos encontrar diversas formas de medirlo, eligiendo finalmente la clasificación utilizada por Orlitzky et al. (2003) ya que es el estudio de referencia en esta problemática, diferenciando por tanto entre medidas contables, de mercado o perceptuales (a través de encuestas).

Por último, dada la influencia que la cultura ejerce sobre la RSC (Waldman et al., 2006), el país en el que se realiza el estudio puede tener influencia en el resultado de la relación. Por ello, vamos a utilizar para esta variable cuatro clasificaciones distintas: (a) el continente al que pertenece el país, (b) el Índice de Competitividad Responsable (principiantes, afirmadores, cumplidores o innovadores $)^{3}$, (c) los ingresos per cápita de cada país según el Banco Mundial en 2007 (altos, medios, bajos) ${ }^{4}$, y (d) el sistema jurídico que rige en cada país (anglosajón, continental o mixto) ${ }^{5}$.

\section{Resultados y análisis}

Los resultados del meta-análisis realizado aparecen resumidos en las tablas 2 y 3 , que se presentan seguidamente, indicando el número de muestras independientes utilizadas en cada análisis $(\mathrm{K})$, el tamaño muestral total $(\mathrm{N})$, el tamaño efecto $(\mathrm{r})$, su intervalo de confianza correspondiente, la pruebas de heterogeneidad con la regla del $75 \%\left(\mathrm{~S}_{2 \mathrm{e}} / \mathrm{S}_{2 \mathrm{r}}\right.$ $\geq 75]$ ) y el coeficiente $\mathrm{Q}$ de homogeneidad, y por último, la prueba de diferencia de medias (F de Ficher-Snedecord).

La Tabla 2 muestra los resultados de la relación general entre RSC y RF. Aunque no podemos extraer conclusiones acerca de la dirección que tiene la causalidad debido a la herramienta utilizada para el análisis, sí podemos hacerlo en cuanto a la influencia positiva o negativa que tienen una sobre otra.

Como puede observarse en la primera fila, los datos obtenidos indican la existencia de una relación positiva entre el RSC y el RF, con un tamaño del efecto de 0.0589 (estadísticamente significativo). Sin embargo, debido a la elevada variabilidad entre las correlaciones de los estudios, no podemos aceptar la hipótesis de homogeneidad de los resultados, lo que sugiere la conveniencia de estudiar los efectos moderadores. Con este objeto, agruparemos los estudios que posean características similares utilizando para ello las variables descritas en el apartado 3.3.

Cuando consideramos las diferentes dimensiones de la RSC según el Enfoque Triple Bottom Line, observamos que aquellos trabajos que utilizan medidas globales de RSC ofrecen lógicamente resultados muy similares a los obtenidos cuando estudiamos nuestra muestra en su totalidad. Sin embargo, los resultados derivados de centrarnos en el estudio de las dimensiones social y medioambiental difieren de los anteriores.

Especialmente relevantes dentro de este grupo, son los resultados del análisis de los trabajos que se centran en los aspectos medioambientales (estadísticamente significativo al $10 \%$ ), ya que muestran una variación debida al azar mucho menor. Este hecho se puede justificar porque las medidas de responsabilidad medioambiental están mucho más estandarizadas, ya que existen regulaciones en todos los países que les obligan a informar sobre la influencia que su actividad tiene sobre el medioambiente. 
Tabla 2. Resultados del Meta-análisis.

\begin{tabular}{|c|c|c|c|c|c|c|c|c|}
\hline \multirow[b]{2}{*}{$\mathrm{RSC} \leftrightarrow \mathrm{RF}$} & \multirow{2}{*}{$\frac{\mathrm{K}}{86}$} & \multirow{2}{*}{$\frac{\mathrm{N}}{30849}$} & \multirow{2}{*}{$\frac{r}{0.0589^{*}}$} & \multicolumn{2}{|c|}{$\begin{array}{c}\text { Intervalo de } \\
\text { confianza (95\%) }\end{array}$} & \multirow{2}{*}{$\frac{75 \%}{14.60 \%}$} & \multirow{2}{*}{$\frac{\mathrm{Q}}{591.881 * * *}$} & \multirow[t]{2}{*}{$\begin{array}{l}\text { Diferencia } \\
\text { medias }(\mathrm{F})\end{array}$} \\
\hline & & & & 0.0102 & 0.1076 & & & \\
\hline $\mathrm{RSC} \leftrightarrow \mathrm{RF}$ Países & 69 & 22672 & $0.0599 *$ & 0.0013 & 0.1186 & $15.17 \%$ & $569.354 * * *$ & \\
\hline \multicolumn{9}{|l|}{ DIMENSIÓN } \\
\hline $\mathrm{RSC}$ & 39 & 20496 & $0.0547 *$ & 0.0081 & 0.1013 & $23.63 \%$ & $168.270 * * *$ & $35.17 * * *$ \\
\hline Social & 31 & 4994 & 0.0796 & -0.0231 & 0.1824 & $8.34 \%$ & $367.049 * * *$ & \\
\hline Medioambiental & 20 & 5472 & $0.0569 \dagger$ & -0.0024 & 0.1162 & $62.63 \%$ & $31.728 *$ & \\
\hline Filantropía & 6 & 2137 & 0.0559 & -0.2876 & 0.3993 & $15.39 \%$ & $38.740 * * *$ & \\
\hline \multicolumn{9}{|l|}{ VALORACIÓN } \\
\hline Disclosure & 34 & 13591 & $0.0683 * *$ & 0.0009 & 0.1358 & $20.08 \%$ & $167.785 * * *$ & $30.28 * * *$ \\
\hline Compromiso & 40 & 10430 & 0.0599 & -0.0181 & 0.1378 & $11.06 \%$ & $368.126 * * *$ & \\
\hline Cantidades & 7 & 2491 & 0.0361 & -0.2611 & 0.3333 & $16.51 \%$ & $42.283 * * *$ & \\
\hline Índice bursátiles & 6 & 4805 & $0.0383 * *$ & 0.0153 & 0.0613 & $100 \%$ & $14.508^{*}$ & \\
\hline \multicolumn{9}{|c|}{ RENDIMIENTO FINANCIERO } \\
\hline Contables & 55 & 24942 & $0.0663 * *$ & 0.0045 & 0.1281 & $29.17 \%$ & $190.284 * * *$ & $60.40 * * *$ \\
\hline Mercado & 29 & 17148 & 0.0454 & -0.0286 & 0.1194 & $12.74 \%$ & $226.628 * * *$ & \\
\hline Perceptuales & 24 & 3739 & 0.0735 & -0.0556 & 0.2026 & $6.78 \%$ & $350.098 * * *$ & \\
\hline \multicolumn{9}{|l|}{ CONTINENTES } \\
\hline Europa & 23 & 5074 & 0.0551 & -0.0437 & 0.1538 & $19.44 \%$ & $117.619 * * *$ & $41.70^{* * *}$ \\
\hline América & 16 & 6601 & 0.0457 & -0.0315 & 0.1228 & $41.95 \%$ & $37.979 * * *$ & \\
\hline Asia & 23 & 10008 & 0.0871 & -0.0359 & 0.2100 & $8.42 \%$ & $280.693 * * *$ & \\
\hline Oceanía & 6 & 839 & -0.0005 & -0.0935 & 0.0926 & $17.74 \%$ & $33.831 * * *$ & \\
\hline \multicolumn{9}{|c|}{ INGRESOS SEGÚN EL BANCO MUNDIAL } \\
\hline Altos & 39 & 12919 & 0.0550 & -0.0122 & 0.1222 & $13.86 \%$ & $279.585 * * *$ & $28.57 * * *$ \\
\hline Medios & 20 & 7417 & 0.0464 & -0.0473 & 0.1401 & $24.42 \%$ & $85.624 * * *$ & \\
\hline Bajos & 5 & 673 & 0.3614 & -0.2680 & 0.9908 & $2.18 \%$ & $138.663 * * *$ & \\
\hline \multicolumn{9}{|c|}{ ÍNDICE DE COMPETITIVIDAD SOSTENIBLE } \\
\hline Innovador & 31 & 10522 & 0.0264 & -0.0469 & 0.0997 & $17.34 \%$ & $178.539 * * *$ & $17.44 * * *$ \\
\hline Afirmador & 14 & 5565 & 0.0722 & -0.0025 & 0.1469 & $30.34 \%$ & $48.926 * * *$ & \\
\hline Cumplidor & 12 & 1963 & 0.1123 & -0.1099 & 0.3344 & $5.50 \%$ & $212.837 * * *$ & \\
\hline Principiantes & 6 & 2959 & 0.0843 & -0.1158 & 0.2845 & $14.54 \%$ & $40.675 * * *$ & \\
\hline \multicolumn{9}{|c|}{ SISTEMA JURÍDICO } \\
\hline Anglosajón & 24 & 8450 & 0.0465 & -0.0116 & 0.1047 & $31.39 \%$ & $76.135 * * *$ & $23.64 * * *$ \\
\hline Civil & 17 & 2589 & 0.0132 & -0.1236 & 0.1499 & $8.70 \%$ & $195.409 * * *$ & \\
\hline Mixto & 23 & 10008 & 0.0871 & -0.0359 & 0.2100 & $8.42 \%$ & $280.693 * * *$ & \\
\hline
\end{tabular}

$* * *<0.005, * *<0.01, *<0.05, \dagger<0.1$.

En lo referente a las acciones sociales así como a las donaciones o acciones filantrópicas, los resultados no son estadísticamente relevantes, debido probablemente en el segundo caso al número limitado de observaciones disponibles. 
Por tanto, se acepta la Hipótesis sobre la Moderación basada en el Enfoque de la Triple Bottom Line $\left(\mathrm{H}_{2}\right)$, ya que las acciones medioambientales destacan por ser las únicas que muestran una relación entre RSC y RF significativa. Respecto a las acciones filantrópicas, no podemos llegar a conclusión alguna debido a la escasez de observaciones.

En referencia a los grupos creados en función de cómo valoren las empresas sus políticas de RSC, destaca el grupo en el que para ello se crean índices ponderados basados en si las empresas revelan o no información (disclosure) sobre determinadas cuestiones de $\operatorname{RSC}(\mathrm{r}=0.0683)$.

$\mathrm{Si}$ los trabajos valoran la RSC en función del compromiso que las empresas reporten sobre estas acciones, la heterogeneidad detectada es muy elevada $(\mathrm{Q}=368.3610)$, lo cual se justifica por la subjetividad de dicha medida, ya que cada empresa valora según su perspectiva y es difícil encontrar una uniformidad de criterio.

También parece existir cierto consenso en los trabajos que valoran que una empresa es socialmente responsable si está incluida en determinados índices bursátiles ligados a la RSC (coeficiente $r=0.0383$ ), ya que la heterogeneidad detectada es muy pequeña y la prueba de la varianza nos indica que la variabilidad es muy reducida, aunque el número de observaciones no es muy elevado. A pesar de la homogeneidad que existe en los resultados, la literatura especializada (Cho et al., 2012) plantea ciertas deficiencias en esta forma de valorar la RSC de las empresas, ya que las condiciones para estar incluidos no sólo dependen de lo socialmente responsables que sean las empresas, y, consecuentemente empresas con un comportamiento socialmente responsable quedan excluidas de todo análisis o categorizadas como " 0 ".

La influencia que la forma de medir el RF tiene sobre la relación es diversa. En cuanto a las variables tradicionalmente utilizadas (contables y de mercado), encontramos que si se mide a través de medidas contables la influencia es significativa $(r=0.0663)$, mientras que si se mide a través de medidas de mercado o perceptuales la relación entre RSC y RF no es significativa.

Tras la introducción en el análisis del país de estudio, el número de muestras independientes se reduce pasando a ser 69, ya que no todos los trabajos tienen definido su ámbito espacial, o bien éste abarca varios países, por lo cual se hace necesaria su eliminación antes de proceder al estudio estadístico, para poder contrastar realmente si tal y como demuestran Waldman et al. (2006), los rasgos culturales propios de los países afectan al desarrollo del concepto de RSC y, por tanto, a su relación con el RF.

Una vez eliminados los estudios referidos a más de un país, tanto el coeficiente de correlación como su significatividad $(0.0599$, p-value 0.05$)$ prácticamente no varían en relación a los que habíamos obtenido considerando la muestra en su conjunto.

Realizados dichos ajustes, nos encontramos que los resultados obtenidos no son estadísticamente significativos, aunque si dejan ver ciertos comportamientos interesantes, ya que podemos observar un descenso (más o menos acusado) de la heterogeneidad en todas las clasificaciones culturales.

Debido a la gran influencia que el trabajo de Usunier et al. (2011) ejerce en el metaanálisis al aportar 16 de las 86 estimaciones (algo más del 18\%), hemos vuelto a realizar el análisis eliminando dichas estimaciones para valorar si los resultados se están viendo afectados o no (Tabla 3).

En primer lugar, la relación RSC-RF muestra una correlación superior a la anterior y mucho más significativa estadísticamente tanto si se incluyen como si se excluyen los trabajos que abarcan más de un país. 
Tabla 3. Meta-análisis excluido el trabajo de Usunier et al. (2011).

\begin{tabular}{|c|c|c|c|c|c|c|c|c|}
\hline & $\mathrm{K}$ & $\mathrm{N}$ & $\mathrm{r}$ & $\begin{array}{r}\text { Interval } \\
\text { confianza }\end{array}$ & $\begin{array}{l}\text { o de } \\
(95 \%)\end{array}$ & $75 \%$ & Q & $\begin{array}{l}\text { Diferencia } \\
\text { medias }(\mathrm{F})\end{array}$ \\
\hline $\mathrm{RSC} \leftrightarrow \mathrm{RF}$ & 71 & 28924 & $0.0697 * * *$ & 0.0238 & 0.1156 & $20.30 \%$ & $424.470 * * *$ & \\
\hline $\begin{array}{c}\mathrm{RSC} \leftrightarrow \mathrm{RF} \\
\text { Países }\end{array}$ & 53 & 21019 & $0.0757 * *$ & 0.0188 & 0.1325 & $13.11 \%$ & $399.693 * * *$ & \\
\hline \multicolumn{9}{|l|}{ DIMENSIÓN } \\
\hline RSC & 39 & 20496 & $0.0547 *$ & 0.0081 & 0.1013 & $23.63 \%$ & $168.270 * * *$ & \multirow[t]{4}{*}{$48.41 * * *$} \\
\hline Social & 15 & 3189 & $0.1885 * * *$ & 0.0759 & 0.3011 & $9.19 \%$ & $151.760 * * *$ & \\
\hline Medioambiental & 20 & 5472 & $0.0569 \dagger$ & 0.0071 & 0.1067 & $62.63 \%$ & $31.728 *$ & \\
\hline Filantropía & 6 & 2137 & 0.0559 & -0.2876 & 0.3993 & $15.39 \%$ & $38.740 * * *$ & \\
\hline \multicolumn{9}{|l|}{ VALORACIÓN } \\
\hline Disclosure & 34 & 13591 & $0.0683 * *$ & 0.0009 & 0.1358 & $20.08 \%$ & $167.785 * * *$ & \multirow[t]{4}{*}{$37.21 * * *$} \\
\hline Compromiso & 24 & 8625 & $0.0960 * *$ & 0.0253 & 0.1666 & $12.24 \%$ & $192.413 * * *$ & \\
\hline Cantidades & 7 & 2491 & 0.0361 & -0.2611 & 0.3333 & $16.51 \%$ & $42.283^{* * *}$ & \\
\hline Índice bursátiles & 6 & 4805 & $0.0383 * *$ & 0.0153 & 0.0613 & $100 \%$ & 2.813 & \\
\hline \multicolumn{9}{|c|}{ RENDIMIENTO FINANCIERO } \\
\hline Contables & 55 & 24942 & $0.0663 * *$ & 0.0045 & 0.1281 & $29.17 \%$ & $190.284 * * *$ & \multirow[t]{3}{*}{$81.59 * * *$} \\
\hline Mercado & 29 & 17148 & 0.0454 & -0.0286 & 0.1194 & $12.74 \%$ & $226.628 * * *$ & \\
\hline Perceptuales & 8 & 1934 & $0.2473 * *$ & 0.0698 & 0.4249 & $17.87 \%$ & $118.358 * * *$ & \\
\hline \multicolumn{9}{|l|}{ CONTINENTES } \\
\hline Europa & 17 & 4491 & $0.0968^{*}$ & 0.0182 & 0.1755 & $82.25 \%$ & 20.282 & \multirow[t]{4}{*}{$28.15^{* * *}$} \\
\hline América & 12 & 6103 & 0.0519 & -0.0259 & 0.1297 & $47.33 \%$ & $25.216^{* *}$ & \\
\hline Asia & 20 & 9785 & 0.0950 & -0.0199 & 0.2100 & $8.48 \%$ & $231.693 * * *$ & \\
\hline Oceanía & 4 & 640 & -0.0005 & -0.1954 & 0.1944 & $12.93 \%$ & $30.937 * * *$ & \\
\hline \multicolumn{9}{|c|}{ INGRESOS SEGÚN EL BANCO MUNDIAL } \\
\hline Altos & 30 & 11465 & $0.0727 *$ & 0.0117 & 0.1338 & $17.10 \%$ & $173.606^{* * *}$ & \multirow[t]{3}{*}{$47.77 * * *$} \\
\hline Medios & 16 & 7083 & 0.0535 & -0.0181 & 0.1252 & $41.52 \%$ & $50.294 * * *$ & \\
\hline Bajos & 3 & 574 & 0.4979 & -0.0817 & 1.0774 & $2.57 \%$ & $65.967 * * *$ & \\
\hline \multicolumn{9}{|c|}{ ÍNDICE DE COMPETITIVIDAD SOSTENIBLE } \\
\hline Innovador & 22 & 9068 & 0.0442 & -0.0172 & 0.1056 & $26.17 \%$ & $83.728 * * *$ & \multirow[t]{4}{*}{$25.64 * * *$} \\
\hline Afirmador & 12 & 5415 & $0.0785 *$ & 0.0006 & 0.1564 & $37.70 \%$ & $31.439 * * *$ & \\
\hline Cumplidor & 9 & 1703 & 0.1698 & -0.0711 & 0.4106 & $5.26 \%$ & $161.438 * * *$ & \\
\hline Principiantes & 5 & 2936 & 0.0800 & -0.0418 & 0.2019 & $14.62 \%$ & $33.773^{* * *}$ & \\
\hline \multicolumn{9}{|c|}{ SISTEMA JURÍDICO } \\
\hline Anglosajón & 18 & 7450 & 0.0474 & -0.0240 & 0.1188 & $25.49 \%$ & $70.293 * * *$ & \multirow[t]{3}{*}{$35.42 * * *$} \\
\hline Civil & 10 & 1887 & $0.1143 \dagger$ & -0.0199 & 0.2486 & $20.36 \%$ & $81.342 * * *$ & \\
\hline Mixto & 20 & 9785 & 0.0950 & -0.0199 & 0.2100 & $8.48 \%$ & $231.693 * * *$ & \\
\hline
\end{tabular}

$* * *<0.005, * *<0.01, *<0.05, \uparrow<0.1$.

Por otro lado, aquellos trabajos que se centran en el estudio de la dimensión social de la RSC, nos muestran una gran influencia entre las variables del estudio $(r=0.1885)$, aunque la heterogeneidad derivada sigue siendo elevada. Este hecho puede explicarse por 
un doble motivo: la gran cantidad de indicadores diferentes que se incluyen en la dimensión social de la RSC y que no existe un consenso en cuanto los instrumentos de medida utilizados en cada uno de los trabajos.

Otro cambio significativo respecto al análisis anterior viene derivado de aquellos trabajos que valoran la RSC teniendo en cuenta el compromiso de las empresas con dichas políticas $(0.0960$, p-value 0.01$)$. También resulta relevante la correlación obtenida $(\mathrm{r}=0.2473)$ si se mide el RF por medio de encuestas, ya que resulta muy significativa $(0.01)$.

Finalmente, los mayores cambios se muestran en el efecto que el ámbito espacial tiene sobre la relación RSC-RF, ya que se identifican influencias significativas. De este modo, aquellos países europeos y/o con ingresos altos y/o clasificados como afirmadores y/o con un sistema jurídico civil son aquellos en los que la relación entre la RSC y el RF es significativa.

\section{Conclusiones}

Al inicio de este trabajo planteamos como objetivo profundizar en el conocimiento de la relación entre RSC y RF, a través del estudio del papel moderador que determinadas variables pudieran ejercer sobre ella. Para ello, partiendo de los resultados de los trabajos que anteriormente han estudiado dicha cuestión, obtuvimos en una muestra de 70 artículos (86 muestras independientes), cuyo horizonte temporal abarca desde el año 2000 al año 2012.

Tras el análisis de los resultados obtenidos en el meta-análisis, podemos concluir que existe relación entre las políticas de RSC y el RF de las empresas, siendo de carácter positivo y significativa. En esta línea, los resultados obtenidos presentados anteriormente, nos permiten afirmar que las acciones consideradas para evaluar la RSC (social, medioambiental, filantrópica), la forma en la que los investigadores evalúan esas políticas de $\mathrm{RSC}$, el modo en el que se informa sobre el RF de la empresa; afectan a la relación existente entre estas dos variables y, por tanto, moderan dicha relación.

En este sentido, serían las políticas medioambientales las que nos muestran una relación más significativa y homogénea. Adicionalmente, la valoración de las acciones socialmente responsables a través de la información revelada por las empresas en sus informes, junto con la inclusión de las empresas en los índices de sostenibilidad, presentan también una relación significativa entre la RSC y el RF. Refiriéndonos al RF, las medidas contables predicen de forma más homogénea y significativa dichas acciones.

Por último, tras la observación conjunta de los resultados de las diferentes clasificaciones culturales, aunque la evidencia estadística encontrada no es suficientemente robusta, hay indicios suficientes de que la cultura de los países afecta y modera dicha relación.

Dichos resultados invitan a estudiar el efecto que la cultura nacional tiene sobre la relación con mayor profundidad a través de una clasificación que abarque de forma conjunta un mayor número de características culturales que puedan reflejar las diferencias entre los grupos.

En cuanto a las limitaciones derivadas del estudio, debemos señalar la elevada heterogeneidad que existe aunque esta se ha ido reduciendo, en algunos casos de forma considerable, con la agrupación de los casos por la introducción de variables moderadoras de la relación entre RSC y el RF. Adicionalmente, es necesario resaltar que existen países en los cuales no se ha estudiado de forma independiente la relación RSC-RF y que no han sido incluidos en el estudio. 


\section{Notas}

1. Este hecho se debe a que muchos trabajos científicos, en su mayoría con resultados "negativos" (aquellos que no hallan diferencias significativas o con resultados en contra de la hipótesis de estudio o de lo habitualmente establecido), nunca llegan a publicarse, tardan más en hacerlo o son menos citados en otras publicaciones.

2. SSRN (Social Science Research Network) es una base de datos web que se dedica a la rápida difusión en todo el mundo de la investigación en ciencias sociales. Los autores pueden subir sus trabajos académicos directamente. En ella podemos encontrar en circulación trabajos en una etapa temprana de desarrollo o bien trabajos que se encuentran en proceso de revisión en alguna revista.

3. El índice de competitividad responsable (ICR) utiliza veintiún indicadores procedentes de trece fuentes diferentes. Estos indicadores están organizados en tres subíndices (Impulsores de Políticas, Acción Empresarial y Facilitadores sociales) conteniendo cada uno de ellos siete indicadores, y según el cual se divide a los países en cuatro grupos: Principiantes, Afirmadores, Cumplidores e Innovadores. "El estado de la competitividad responsable" (2007).http://www.accountability.org/ images/content/1/0/108/El\%20Estado\%20de\%20la\%20Competitividad\%20Responsable\%202007 .pdf

4. Derivados también del documento "El estado de la competitividad responsable" (2007), en el cual se divide a los países en tres grupos: ingresos altos, medios y bajos.

5. http://www.juriglobe.ca/esp/sys-juri/index-alpha.php.

\section{Referencias bibliográficas}

Aguilera, R., Rupp, D. E., Williams, C. A., \& Ganapathi, J. (2007). Putting the S back in corporate social responsibility: A multilevel theory of social change in organizations. Academy of Management Review, 32(3), 836-863. doi:10.5465/AMR.2007.25275678

Ahmad, N. H., \& Ramayah, T. (2012). Does the notion of 'Doing Well by Doing Good' prevail among entrepreneurial ventures in a developing nation?. Journal of Business Ethics, 106(4), 479-490. doi:10.1007/s10551-011-1012-9

Ali, I., Rehman, K. U., Ali, S. I., Yousaf, J., \& Zia, M. (2010). Corporate social responsibility influences, employee commitment and organizational performance. African Journal of Business Management, 4(12), 2796-2801.

Allouche, J., \& Laroche, P. (2005). A meta-analytical investigation of the relationship between Corporate Social and Financial Performance. Revue De Gestion Des Resources Humaines, 57, $18-41$.

Al-Tuwaijri, S.A., Christensen, T. E., \& Hughes, K. E. (2004). The relations among environmental disclosure, environmental performance, and economic performance: A simultaneous equations approach. Accounting, Organizations and Society, 29, 447-471. doi:10.1016/S0361-3682(03) 00032-1

Andayani, W., Atmini, S., \& Kamau, J. (2008). Corporate Social Responsibility, Good Corporate Governance and the Intellectual property: An external strategy of the management to increase the company's value. National Conference on Management Research, Makassar.

Apostolakau, A., \& Jackson, G. (2009). Corporate Social Responsibility in Western Europe: An institutional mirror or substitute (University of Bath, School Management Working Paper Series).

Aras, G., Aybars, A., \& Kutlu, O. (2010). Managing corporate performance: Investigating the relationship between corporate social responsibility and financial performance in emerging markets. International Journal of Productivity and Performance Management, 59(3), 229254. doi:10.1108/17410401011023573

Auamnoy, T., \& Areepium, N. (2011). PRM8 could corporate social responsibility predict pharmaceutical corporate financial performance?. Value in Health, 14, A422-. doi:10.1016/j. jval.2011.08.1039

Bartkus, B., Glassman, M., \& McAfee, B. (2006). Mission statement quality and financial performance. European Management Journal, 24(1), 86-94. doi:10.1016/j.emj.2005.12.010

Baughn, C. C., Body, N., \& McIntosh, J. C. (2007). Corporate social and environmental responsibility in Asian countries and other geographical regions. Corporate Social Responsibility and Environmental Management, 14(4), 189-205. doi:10.1002/csr.160 
Bedi, H. (2009). Financial performance and social responsibility: Indian scenario. Available at SSRN 1496291.

Berrone, P., Surroca, J., \& Tribó, J. A. (2007). Corporate ethical identity as a determinant of firm performance: A test of the mediating role of stakeholder satisfaction. Journal of Business Ethics, 76, 35-53. doi:10.1007/s10551-006-9276-1

Beurden, P., \& Gössling, T. (2008). The worth of values - A literature review on the relation between corporate social and financial performance. Journal of Business Ethics, 82, 407-424. doi:10.1007/s10551-008-9894-x

Brammer, S., \& Millington, A. (2005). Corporate reputation and philanthropy: An empirical analysis. Journal of Business Ethics, 61(1), 29-44. doi:10.1007/s10551-005-7443-4

Burke, L., \& Logsdon, J. M. (1996). How corporate social responsibility pays off. Long Range Planning, 29(4), 495-502. doi:10.1016/0024-6301(96)00041-6

Cabeza-García, L., Martínez-Campillo, A., \& Marbella-Sánchez, F. (2010). Análisis de la relación entre los resultados sociales y económicos de las cajas de ahorros españolas: un caso de responsabilidad social corporativa. Innovar, 20(37), 33-46.

Carroll, A. (1979). A three dimensional conceptual model of corporate performance. Academy of Management Review, 4(4), 497-505.

Carroll, A. (1991). The pyramid of corporate social responsibility: Toward the moral management of organizational stakeholders. Business Horizons, 34(4), 39-48. doi:10.1016/0007-6813(91) 90005-G

Chih, H. L., Chih, H. H., \& Chen, T. Y. (2010). On the determinants of corporate social responsibility: International evidence on the financial industry. Journal of Business Ethics, 93(1), 115135. doi:10.1007/s10551-009-0186-x

Cho, C. H., Guidry, R. P., Hageman, A. M., \& Patten, D. M. (2012). Do actions speak louder than words? An empirical investigation of corporate environmental reputation. Accounting, Organizations and Society, 37(1), 14-25. doi:10.1016/j.aos.2011.12.001

Choi, J. S., Kwak, Y. M., \& Choe, C. (2010). Corporate social responsibility and corporate financial performance: Evidence from Korea. Australian Journal of Management, 35(3), 291-311. doi: $10.1177 / 0312896210384681$

Choi, T. H., \& Jung, J. (2008). Ethical commitment, financial performance, and valuation: An empirical investigation of Korean companies. Journal of Business Ethics, 81(2), 447-463. doi:10.1007/s10551-007-9506-1

Clarkson, P. M., Li, Y., Richardson, G. D., \& Vasvari, F. P. (2008). Revisiting the relation between environmental performance and environmental disclosure: An empirical analysis. Accounting, Organizations and Society, 33(4-5), 303-327. doi:10.1016/j.aos.2007.05.003

Clemens, B. (2006). Economic incentives and small firms: Does it pay to be green?. Journal of Business Research, 59, 492-500. doi:10.1016/j.jbusres.2005.08.006

Cohen, J. (1969). Statistical power analysis for the behavioural sciences. New York: Academic Press.

Cormier, D., \& Magnan, M. (2003). Environmental reporting management: A continental European perspective. Journal of Accounting and Public Policy, 22(1), 43-62. doi:10.1016/S0278-4254 (02)00085-6

Crane, A., \& Matten, D. (2007). Business ethics: Managing corporate citizenship and sustainability in the age of globalization. USA: Oxford University Press.

Crisóstomo, V., Freire, F., \& Cortes de Vasconcellos, F. (2011). Corporate social responsibility, firm value and financial performance in Brazil. Social Responsibility Journal, 7(2), 295-309. doi: $10.1108 / 17471111111141549$

Dahlsrud, A. (2008). How corporate social responsibility is defined: An analysis of 37 definitions. Corporate Social-Responsibility and Environmental Management, 15(1), 1-13. doi:10.1002/ csr. 132

Dam, L., \& Scholtens, B. (2012). Does ownership type matter for corporate social responsibility?. Corporate Governance: An International Review, 20(3), 233-252. doi:10.1111/j.14678683.2011.00907.x

Da Silva-Monteiro, S. M., \& Aibar-Guzmán, B. (2010). Determinants of environmental disclosure in the annual reports of large companies operating in Portugal. Corporate Social Responsibility and Environmental Management, 17(4), 185-204. doi:10.1002/csr.197

De la Cuesta, M. (2004). El porqué de la Responsabilidad Social Corporativa. Boletín Económico De ICE, 2813, 45-58. 
Dunn, P., \& Sainty, B. (2009). The relationship among board of director characteristics, corporate social performance and corporate financial performance. International Journal of Managerial Finance, 5(4), 407-423. doi:10.1108/17439130910987558

Eisenhardt, K. M. (1989). Agency theory: An assessment and review. The Academy of Management Review, 14(1), 57-74.

Elkington, J. (1998). Partnerships from cannibals with forks: The triple bottom line of 21 st century business. Environmental Quality Management, 8(1), 37-51. doi:10.1002/tqem.3310080106

Elsayed, K. K., \& Paton, D. (2005). The impact of environmental performance on firm performance: Static and dynamic panel data evidence. Structural Change and Economic Dynamics, 16, 395412. doi:10.1016/j.strueco.2004.04.004

Fauzi, H. (2010). Corporate social and financial performance: Empirical evidence from American companies. SSRN.

Fauzi, H., Hussain, M. M., Rahman, A. A., \& Priyanto, A. A. (2009). Corporate social performance of Indonesian state-owned and private companies. SSRN.

Fauzi, H., Mahoney, L., \& Hahaman, A. A. (2007). Institutional ownership and corporate social performance: Empirical evidence from Indonesian companies. Issues in Social and Environmental Accounting, 1(2), 334-347.

Fifka, M. S. (2013). Corporate responsibility reporting and its determinants in comparative perspective - A review of the empirical literature and a meta-analysis. Business Strategy and the Environment, 22(1), 1-35. doi:10.1002/bse.729

Fowler, S. J., \& Hope, C. (2007). A critical review of sustainable business indices and their impact. Journal of Business Ethics, 76(3), 243-252. doi:10.1007/s10551-007-9590-2

Freeman, R. E. (1984). Strategic management: A stakeholder approach. Boston, MA: Pitman.

Friedman, M. (1970). The social responsibility of business is to increase its profits. New York Times, 13(September) 122-126.

Galbreath, J. (2006). Does primary stakeholder management positively affect the bottom line?: Some evidence from Australia. Management Decision, 44(8), 1106-1121. doi:10.1108/ 00251740610690649

Garay, L., \& Font, X. (2012). Doing good to do well? Corporate social responsibility reasons, practices and impacts in small and medium accommodation enterprises. International Journal of Hospitality Management, 31(2), 329-337. doi:10.1016/j.ijhm.2011.04.013

Garcia-Castro, R., Ariño, M., \& Canela, M. A. (2010). Does social performance really lead to financial performance? Accounting for endogeneity. Journal of Business Ethics, 92, 107-126. doi:10.1007/s10551-009-0143-8

García-Meca, E., \& Sánchez-Ballesta, J. P. (2006). Un estudio meta-analítico de los factores determinantes de la revelación de información. Spanish Journal of Finance and Accounting / Revista Española de Financiación y Contabilidad, 35, 761-788. doi:10.1080/ 02102412.2006.10779605

García-Meca, E., \& Sánchez-Ballesta, J. P. (2010). The association of board independence and ownership concentration with voluntary disclosure: A meta-analysis. European Accounting Review, 19(3), 603-627. doi:10.1080/09638180.2010.496979

Garriga, E., \& Melé, D. (2004). Corporate social responsibility theories: Mapping the territory. Journal of Business Ethics, 53, 51-71. doi:10.1023/B:BUSI.0000039399.90587.34

Godos-Díez, J. L., Fernández-Gago, R., \& Cabeza-García, L. (2012). Propiedad y control enla puesta en práctica de la RSC. Cuadernos de Economía y Dirección de la Empresa, 15(1), 1-11. 10.1016/j.cede.2011.06.002

Goll, I., \& Rasheed, A. (2004). The moderating effect of environmental munificence and dynamism on the relationship between discretionary social responsibility and financial performance. Journal of Business Ethics, 49, 41-54. doi:10.1023/B:BUSI.0000013862.14941.4e

Gómez, F. (2008). Responsabilidad Social Corporativa y Performance Financiero: Treinta y cinco años de investigación empírica en busca de un consenso. Principios, 11, 5-22.

Gray, R., Javad, M., Power, D. M., \& Sinclair, C. D. (2001). Social and environmental disclosure and corporate characteristics: A research note and extension. Journal of Business Finance \& Accounting, 28(3-4), 327-356. doi:10.1111/1468-5957.00376

Guenster, N., Bauer, R., Derwall, J., \& Koedijk, K. (2011). The economic value of corporate ecoefficiency. European Financial Management, 17, 679-704. doi:10.1111/j.1468036X.2009.00532.x 
Hackston, D., \& Milne, M. J. (1996). New some determinants of social and environmental disclosures in New Zealand companies accounting. Accounting, Auditing \& Accountability Journal, 9(1), 77-108. doi:10.1108/09513579610109987

Hamid, K., Akash, R. S. I., Asghar, M., \& Ahmad, S. (2011). Corporate social performance, financial performance and market value behavior: An information asymmetry perspective. African Journal of Business Management, 5(15), 6342-6349. doi:10.5897/AJBM10.1102

Haniffa, R. M., \& Cooke, T. E. (2005). The impact of culture and governance on corporate social reporting. Journal of Accounting and Public Policy, 24(5), 391-430. doi:10.1016/j. jaccpubpol.2005.06.001

He, Y., Tian, Z., \& Chen, Y. (2007). Performance implications of nonmarket strategy in China. Asia Pacific Journal of Management, 24, 151-169. doi:10.1007/s10490-006-9030-3

Hedge, L. V., \& Olkin, I. (1985). Statistical methods for meta-analysis. New York: Academic Press.

Hubbard, G. (2009). Measuring organizational performance: Beyond the triple bottom line. Business Strategy and the Environment, 18(3), 177-191. doi:10.1002/bse.564

Hughes, S. B., Anderson, A., \& Golden, S. (2001). Corporate environmental disclosures: are they useful in determining environmental performance?. Journal of Accounting and Public Policy, 20, 217-240. doi:10.1016/S0278-4254(01)00031-X

Hull, C. E., \& Rothenberg, S. (2008). Firm performance: the interactions of corporate social performance with innovation and industry differentiation. Strategic Management Journal, 29 (7), 781-789. doi:10.1002/smj.675

Hunter, J. E., \& Schmidt, F. L. (1990). Methods of Meta-analysis. Beverly Hills, CA: Sage.

Husted, B. W., \& Allen, D. W. (2000). Is it ethical to use ethics as a strategy. Journal of Business Ethics, 27(1/2), 21-31. doi:10.1023/A:1006422704548

Inoue, Y., \& Lee, S. (2011). Effects of different dimensions of corporate social responsibility on corporate financial performance in tourism-related industries. Tourism Management, 32(4), 790804. doi:10.1016/j.tourman.2010.06.019

Jamali, D., \& Mirshak, R. (2007). Corporate Social Responsibility (CSR): Theory and practice in a developing country context. Journal of Business Ethics, 72(3), 243-262. doi:10.1007/s10551006-9168-4

Kirkham, J. J., \& Dwan, K. M. (2010). The impact of outcome reporting bias in randomized controlled trials on a cohort of systematic reviews. British Medical Journal, 2010, 340-365. doi:10.1136/bmj.c365

Lanis, R., \& Richardson, G. (2012). Corporate social responsibility and tax aggressiveness: Anempirical analysis. Journal of Accounting and Public Policy, 31(1), 86-108. doi:10.1016/j. jaccpubpol.2011.10.006

Lee, S., \& Park, S. Y. (2010). Financial impacts of socially responsible activities on airline companies. Journal of Hospitality \& Tourism Research, 34(2), 185-203. doi:10.1177/ 1096348009349822

Li, W., \& Zhang, R. (2010). Corporate social responsibility, ownership structure, and political interference: Evidence from China. Journal of Business Ethics, 96(4), 631-645. doi:10.1007/ s10551-010-0488-z

Lin, C. H., Yang, H. L., \& Liou, D. Y. (2009). The impact of corporate social responsibility on financial performance: Evidence from business in Taiwan. Technology in Society, 31, 56-63. doi:10.1016/j.techsoc.2008.10.004

Lipsey, M., \& Wilson, D. (2001). Practical meta-analysis. Applied Social Research Methods, 40.

Liu, X., \& Anbumozhi, V. (2009). Determinant factors of corporate environmental information disclosure: An empirical study of Chinese listed companies. Journal of Cleaner Production, 17(6), 593-600. doi:10.1016/j.jclepro.2008.10.001

López, M. V., Garcia, A., \& Rodriguez, L. (2007). Sustainable development and corporate performance: A study based on the Dow Jones sustainability index. Journal of Business Ethics, 75(3), 285-300. doi:10.1007/s10551-006-9253-8

Luo, X., \& Bhattacharya, C. B. (2006). Corporate social responsibility, customer satisfaction and market value. Journal of Marketing, 70, 1-18. doi:10.1509/jmkg.70.4.1

Lyon, D. (2007). Financial performance: The motivation behind CSR reporting (Dissertation).

Makni, R., Francoeur, C., \& Bellavance, F. (2009). Causality between corporate social performance and financial performance: Evidence from Canadian firms. Journal of Business Ethics, 89(3), 409-422. doi:10.1007/s10551-008-0007-7 
Matten, D., \& Moon, J. (2008). Implicit and explicit CSR: A conceptual framework for a comparative understanding of corporate social responsibility. Academy of Management Review, 33(2), 404-424. doi:10.5465/AMR.2008.31193458

McWilliams, A., \& Siegel, D. (2001). Corporate social responsibility: A theory of the firm perspective. Academy of Management Review, 26(1), 117-127. doi:10.5465/ AMR.2001.4011987

Melo, T. (2012). Determinants of corporate social performance: The influence of organizational culture, management tenure and financial performance. Social Responsibility Journal, 8(1), 3347. doi:10.1108/17471111211196557

Melo, T., \& Garrido-Morgado, A. (2012). Corporate reputation: A combination of social responsibility and industry. Corporate Social Responsibility and Environmental Management, 19(1), 1131. doi:10.1002/csr.260

Moneva, J. M., \& Ortas, E. (2010). Corporate environmental and financial performance: A multivariate approach. Industrial Management \& Data Systems, 110(2), 193-210. doi:10.1108/ 02635571011020304

Moore, G. (2001). Corporate social and financial performance: An investigation in the UK supermarket industry. Journal of Business Ethics, 34(3-4), 299-315. doi:10.1023/A:1012537016969

Moroney, R., Windsor, C., \& Aw, Y. T. (2012). Evidence of assurance enhancing the quality of voluntary environmental disclosures: An empirical analysis. Accounting \& Finance, 52(3), 903939. doi:10.1111/j.1467-629X.2011.00413.x

Nakao, Y., Amano, A., Matsumura, K., Genba, K., \& Nakano, M. (2007). Relationship between environmental performance and financial performance: An empirical analysis of Japanese corporations. Business Strategy and the Environment, 16, 106-118. doi:10.1002/bse.476

Nieto, M., \& Fernández, R. (2004). Responsabilidad social corporativa: la última innovación en management. Universia Business Review, primer trimestre 2004, 28-39.

Oh, W. Y., Chang, Y. K., \& Martynov, A. (2011). The effect of ownership structure on corporate social responsibility: Empirical evidence from Korea. Journal of Business Ethics, 104(2), 283 297. doi:10.1007/s10551-011-0912-z

Orlitzky, M., Schmidt, F. L., \& Rynes, S. L. (2003). Corporate social and financial performance: A meta-analysis. Organization Studies, 24, 403-441. doi:10.1177/0170840603024003910

Porter, M. E., \& Van der Linde, C. (1995). Green and competitive: Ending the stalemate. Harvard Business Review, 73(5), 120-134.

Prado, J. M., \& García, I. M. (2011). Entorno cultural y transparencia informativa. AECA, 95, 85-88.

Prado-Lorenzo, J. M., Gallego-Alvarez, I., \& Garcia-Sanchez, I. M. (2009). Stakeholder engagement and corporate social responsibility reporting: The ownership structure effect. Corporate Social Responsibility and Environmental Management, 16(2), 94-107. doi:10.1002/csr.189

Preston, L. E., \& O'Bannon, D. P. (1997). The corporate social-financial performance relationship: A typology and analysis. Business \& Society, 36, 419-429. doi:10.1177/000765039703600406

Prior, D., Surroca, J., \& Tribó, J. A. (2008). Are socially responsible managers really ethical? Exploring the relationship between earnings management and corporate social responsibility?. Corporate Governance: An International Review, 16(3), 160-177. doi:10.1111/j.14678683.2008.00678.x

Purnomo, P. K., \& Widianingsih, L. P. (2012). The influence of environmental performance on financial performance with corporate social responsibility (CSR) disclosure as a moderating variable: Evidence from listed companies in Indonesia. Review of Integrative Business Economic Research, 1(1), 57-69.

Rettab, B., Ben Brik, A. B., \& Mellahi, K. (2009). A study of management perceptions of the impact of corporate social responsibility on organisational performance in emerging economies: The case of Dubai. Journal of Business Ethics, 89(3), 371-390. doi:10.1007/s10551-008-0005-9

Ringov, D., \& Zollo, M. (2007). Corporate responsibility from a socio-institutional perspective. The impact of national culture on corporate social performance. Corporate Governance, 7(4), 476485. doi:10.1108/14720700710820551

Rosenthal, R. (1991). Meta-analytic procedures for social research. Newbury Park, CA: Sage.

Ross, S. A. (1973). The economic theory of agency: The principal's problem. The American Economic Review, 63(2), 134-139.

Ruf, B. M., Muralidhar, K., Brown, R., Janney, J., \& Paul, K. (2001). An empirical investigation of the relationship between change in corporate social performance and financial performance: A 
stakeholder theory perspective. Journal of Business Ethics, 32, 143-156. doi:10.1023/ A: 1010786912118

Sahin, K., Basfirinci, C. S. Y., \& Ozsalih, A. (2011). The impact of board composition on corporate financial and social responsibility performance: Evidence from public-listed companies in Turkey. African Journal of Business Management, 5(7), 2959-2978.

Salama, A. (2005). A note on the impact of environmental performance on financial perfor-mance. Structural Change and Economic Dynamics, 16, 413-421. doi:10.1016/j.strueco.2004.04.005

Salzmann, O., Ionescu-Somers, A., \& Steger, U. (2005). The business case for corporate sustainability: Literature review and research options. European Management Journal, 23, 27-36. doi:10.1016/j.emj.2004.12.007

Sánchez, J. L., \& Sotorrío, L. (2007). The creation of value through corporate reputation. Journal of Business Ethics, 76(3), 335-346. doi:10.1007/s10551-006-9285-0

Sánchez-Meca, J. (2008). Meta-análisis de la investigación, Metodología en la investigación sobre discapacidad. Introducción al uso de las ecuaciones estructurales. VI Simposio Científico SAID, 2008.

Sánchez-Meca, J. (2010). Cómo realizar una revisión sistemática y un meta-análisis. Aula Abierta, $38(2), 53-64$.

Schadewith, H., \& Niskala, M. (2010). Communication via responsibility reporting and its effect on firm value in Finland. Corporate Social Responsibility and Environmental Management, 17, 96106. doi: $10.1002 /$ csr.234

Schmidt, F. L., \& Hunter, J. E. (1977). Development of a general solution to the problem of validity generalization. Journal of Applied Psychology, 62, 529-540. doi:10.1037/0021-9010.62.5.529

Scholtens, B., \& Kang, F. C. (2013). Corporate social responsibility and earnings management: Evidence from Asian economies. Corporate Social Responsibility and Environmental Management, 20(2), 95-112. doi:10.1002/csr.1286

Shen, C. H., \& Chang, Y. (2009). Ambition versus conscience, does corporate social responsibility pay off? The application of matching methods. Journal of Business Ethics, 88(Suppl. S1), 133153. doi:10.1007/s10551-008-9826-9

Singh, J., Salmones Sanchez, M. M., \& Del Bosque, I. R. (2008). Understanding corporate social responsibility and product perceptions in consumer markets: A crosscultural evaluation. Journal of Business Ethics, 80(3), 597-611. doi:10.1007/s10551-007-9457-6

Smith, M. L., \& Glass, G. V. (1977). Meta-analysis of psychotherapy outcome studies. American Psychologist, 32, 752-760. doi:10.1037/0003-066X.32.9.752

Soana, M. G. (2011). The relationship between corporate social performance and corporate financial performance in the banking sector. Journal of Business Ethics, 104(1), 133-148. doi:10.1007/ s10551-011-0894-x

Surroca, J., Tribó, J., \& Waddock, S. (2010). Corporate responsibility and financial performance: The role of intangible resources. Strategic Management Journal, 31, 463-490. doi:10.1002/ smj. 820

Svensson, G., Wood, G., Singh, J., Carasco, E., \& Callaghan, M. (2009). Ethical structures and processes of corporations operating in Australia, Canada, and Sweden: A longitudinal and crosscultural study. Journal of Business Ethics, 86(4), 485-506. doi:10.1007/s10551-008-9860-7

Tagesson, T., Blank, V., Broberg, P., \& Collin, S. O. (2009). What explains the extent and content of social and environmental disclosures on corporate websites: A study of social and environmental reporting in Swedish listed corporations. Corporate Social Responsibility and Environmental Management, 16(6), 352-364. doi:10.1002/csr.194

Toro, D. (2006). El Enfoque estratégico de la Responsabilidad Social Corporativa: una revisión de la literatura académica. Intangible Capital, 14(2), 338-358.

Tsoutsoura, M. (2004). Corporate social responsibility and financial performance. Retrieved from http://escholarship. org/uc/item/111799p2

Usunier, J. C., Furrer, O., \& Furrer-Perrinjaquet, A. (2011). The perceived trade-off between corporate social and economic responsibility: A cross-national study. International Journal of Cross Cultural Management, 11(3), 279-302. doi:10.1177/1470595811413102

Van der Laan, G., Van Ees, H. Y., \& Van Witteloostuijn, A. (2008). Corporate social and financial performance: An extended stakeholder theory, and empirical test with accounting measures. Journal of Business Ethics, 79, 299-310. doi:10.1007/s10551-007-9398-0

Vergalli, S., \& Poddi, L. (2009). Does corporate social responsibility affect the performance of firms? (FEEM Working Paper No. 52.2009). 
Waddock, S. A., \& Graves, S. B. (1997). Corporate social performance-financial performance link. Strategic Management Journal, 18(4), 303-319. doi:10.1002/(SICI)1097-0266(199704) 18:4<303::AID-SMJ869>3.0.CO;2-G

Waldman, D. A., de Luque, M. S., Washburn, N., \& House, R. J. (2006). Cultural and leadership predictors of corporate social responsibility values of top management: A GLOBE study of 15 countries. Journal of International Business Studies, 37, 823-837. doi:10.1057/palgrave. jibs.8400230

Wang, H., \& Qian, C. (2011). Corporate philanthropy and corporate financial performance: The roles of stakeholder response and political access. Academy of Management Journal, 54(6), 11591181. doi:10.5465/amj.2009.0548

Williamson, O. E. (1965). The economics of discretionary behavior: Managerial objectives in a theory of the firm. Chicago, IL: Markham.

Williamson, D., Lynch-Wood, G., \& Ramsay, J. (2006). Drivers of environmental behaviour in manufacturing SMEs and the implications for CSR. Journal of Business Ethics, 67, 317-330. doi:10.1007/s10551-006-9187-1

Wolf, F. M. (1986). Meta-analysis: Quantitative methods for research synthesis. Beverly Hills, CA: Sage.

Wong, E. M., Ormiston, M. E., \& Tetlock, P. E. (2011). The effects of top management team integrative complexity and decentralized decision making on corporate social performance. Academy of Management Journal, 54(6), 1207-1228. doi:10.5465/amj.2008.0762

$\mathrm{Wu}, \mathrm{M}$. L. (2006). Corporate social performance, corporate financial performance and firm size. Journal of American Academy of Business, Cambridge, 8(1), 163-171.

Yang, F. J., Lin, C. W., \& Chang, Y. N. (2010). The linkage between corporate social performance and corporate financial performance. African Journal of Business Management, 4(4), 406-413.

Yong, A. (2008). Cross-cultural comparisons of managerial perceptions on profit. Journal of Business Ethics, 82(4), 775-791. doi:10.1007/s10551-007-9592-0

Yu, V., Ting, H. I., \& Wu, Y. C. (2009). Assessing the greenness effort for European firms: A resource efficiency perspective. Management Decision, 47(7), 1065-1079. doi:10.1108/ 00251740910978304 


\section{Anexo 1}

\begin{tabular}{|c|c|c|c|c|}
\hline Artículo & Periodo estudiado & $\begin{array}{l}\text { Tamaño } \\
\text { muestral }\end{array}$ & $\begin{array}{c}\text { Variable } \\
\text { dependiente }\end{array}$ & Ámbito Espacial \\
\hline Moore (2001) & $1997-2000$ & 8 & - & Reino Unido \\
\hline Cormier \& Magnan (2003) & & 241 & $\mathrm{RSC}$ & Francia \\
\hline Tsoutsoura (2004) & $1996-2000$ & 422 & $\mathrm{RF}$ & EE.UU. \\
\hline Goll \& Rasheed (2004) & 1985-1986 & 62 & $\mathrm{RF}$ & EE.UU. \\
\hline Elsayed \& Paton (2005) & 2004 & 227 & $\mathrm{RF}$ & Reino Unido \\
\hline Salama (2005) & 2000 & 201 & $\mathrm{RF}$ & Reino Unido \\
\hline Haniffa \& Cooke (2005) & 139 & $1996 / 2002$ & $\mathrm{RSC}$ & Malasia \\
\hline Brammer \& Millington (2005) & 2002 & 209 & $\mathrm{RSC}$ & Reino Unido \\
\hline $\begin{array}{l}\text { Bartkus, Glassman, \& McAfee } \\
\text { (2006) }\end{array}$ & 2001 & 56 & $\mathrm{RF}$ & $\begin{array}{l}\text { Europa, Japón y } \\
\text { EE.UU. }\end{array}$ \\
\hline Galbreath (2006) & 2000 & 38 & $\mathrm{RF}$ & Australia \\
\hline Luo \& Bhattacharya (2006) & 2001-2004 & 113 & $\mathrm{RF}$ & \\
\hline Clemens (2006) & 2003 & 76 & $\mathrm{RF}$ & EE.UU \\
\hline $\begin{array}{l}\text { Nakao, Amano, Matsumura, } \\
\text { Genba, \& Nakano (2007) }\end{array}$ & $2002-2003$ & 278 & Ambas & Japón \\
\hline He, Tian,\& Chen (2007) & 2005 & 438 & $\mathrm{RF}$ & China \\
\hline $\begin{array}{l}\text { López , García, \& Rodríguez } \\
\text { (2007) }\end{array}$ & $1998-2004$ & 110 & $\mathrm{RF}$ & \\
\hline Fernández \& Luna (2007) & 2004 & 100 & $\mathrm{RF}$ & \\
\hline $\begin{array}{l}\text { Berrone, Surroca, \& Tribó } \\
\text { (2007) }\end{array}$ & $2002-2003$ & 398 & $\mathrm{RF}$ & 26 países \\
\hline Lyon (2007) & 2004-2005 & 120 & & Nueva Zelanda \\
\hline $\begin{array}{l}\text { Fauzi, Mahoney, \& Hahaman } \\
\text { (2007) }\end{array}$ & 2005 & 324 & $\mathrm{RSC}$ & Indonesia \\
\hline $\begin{array}{l}\text { Clarckson, Li, Richardson, \& } \\
\quad \text { Vasvari (2008) }\end{array}$ & 2003 & 191 & $\mathrm{RSC}$ & EE.UU. \\
\hline Van der Laan et al. (2008) & $1997-2002$ & 734 & $\mathrm{RF}$ & \\
\hline Prior et al. (2008) & $2002-2004$ & 593 & $\mathrm{RSC}$ & 26 países \\
\hline Choi \& Jung (2008) & $2004-2005$ & 248 & $\mathrm{RF}$ & \\
\hline $\begin{array}{l}\text { Andayani, Atmini, \& Kamau } \\
\text { (2008) }\end{array}$ & 2004-2006 & 18 & $\mathrm{RF}$ & Indonesia \\
\hline Liu \& Anbumozhi (2009) & 2006 & 175 & $\mathrm{RSC}$ & China \\
\hline $\begin{array}{l}\text { Tagesson, Blank, Broberg, \& } \\
\text { Collin (2009) }\end{array}$ & $2006-2007$ & 267 & $\mathrm{RSC}$ & Suecia \\
\hline Bedi (2009) & $2007-2008$ & 37 & $\mathrm{RSC}$ & India \\
\hline Dunn \& Sainty (2009) & $2002-2006$ & 104 & $\mathrm{RSC}$ & Canadá \\
\hline $\begin{array}{l}\text { Rettab, Ben Brik, \& Mellahi } \\
\text { (2009) }\end{array}$ & & 280 & $\mathrm{RF}$ & $\begin{array}{l}\text { Emiratos Árabes } \\
\text { Unidos }\end{array}$ \\
\hline $\begin{array}{l}\text { Makni, Francoeur, \& Bellavance } \\
\text { (2009) }\end{array}$ & 2004-2005 & 179 & Ambas & Canadá \\
\hline Shen \& Chang (2009) & $2005-2006$ & 640 & $\mathrm{RF}$ & Taiwán \\
\hline
\end{tabular}


(Continuación).

\begin{tabular}{|c|c|c|c|c|}
\hline Artículo & Periodo estudiado & $\begin{array}{l}\text { Tamaño } \\
\text { muestral }\end{array}$ & $\begin{array}{l}\text { Variable } \\
\text { dependiente }\end{array}$ & Ámbito Espacial \\
\hline Yu, Ting, Wu (2009) & $2002-2007$ & 51 & & Europa \\
\hline Apostolakau \& Jackson (2009) & $2007-2008$ & 248 & $\mathrm{RSC}$ & Europa \\
\hline $\begin{array}{l}\text { Fauzi, Hussain, Rahman, \& } \\
\quad \text { Priyanto (2009) }\end{array}$ & $2001-2004$ & 461 & & Indonesia \\
\hline Lin, Yang, \& Liou (2009) & $2002-2004$ & 33 & $\mathrm{RF}$ & Taiwán \\
\hline Vergalli \& Poddi (2009) & 2001 & 417 & $\mathrm{RF}$ & $\begin{array}{l}\text { EE.UU., Europa } \\
\text { y Japón }\end{array}$ \\
\hline $\begin{array}{l}\text { García-Castro, Ariño, \& Canela. } \\
\text { (2010) }\end{array}$ & $1991 / 2005$ & 658 & $\mathrm{RF}$ & EE.UU \\
\hline Surroca et al. (2010) & $2002-2004$ & 599 & Ambas & $\begin{array}{l}\text { Europa, Norte } \\
\text { América y } \\
\text { Japón }\end{array}$ \\
\hline Yang, Lin, \& Chang (2010) & $2005-2007$ & 150 & Ambas & Taiwán \\
\hline Moneva \& Ortas (2010) & 2004-2007 & 230 & $\mathrm{RF}$ & Europa \\
\hline Chih, Chih, \& Chen (2010) & $2003-2005$ & 520 & $\mathrm{RSC}$ & 34 países \\
\hline Aras, Aybars, \& Kutlu (2010) & $2005-2007$ & 40 & $\mathrm{RSC}$ & Turquía \\
\hline Fauzi (2010) & 2004-2006 & 120 & Ambas & EE.UU. \\
\hline Schadewith \& Niskala (2010) & $2002-2005$ & 236 & $\mathrm{RF}$ & Finlandia \\
\hline $\begin{array}{l}\text { Da Silva Monteiro \& Aibar- } \\
\text { Guzmán (2010) }\end{array}$ & 2002-2004 & 109 & $\mathrm{RSC}$ & Portugal \\
\hline Cabeza-García et al. (2010) & $1992-2005$ & 46 & $\mathrm{RF}$ & España \\
\hline Li \& Zhang (2010) & 2007 & 692 & $\mathrm{RSC}$ & China \\
\hline Choi, Kwak, \& Choe (2010) & $2002-2008$ & 1222 & $\mathrm{RF}$ & Corea \\
\hline $\begin{array}{l}\text { Ali, Rehman, Ali, Yousaf, \& Zia } \\
\text { (2010) }\end{array}$ & & 371 & $\mathrm{RF}$ & Pakistán \\
\hline Lee \& Park (2010) & $1991-2006$ & 41 & $\mathrm{RF}$ & Sin identificar \\
\hline Lima et al. (2011) & $2001-2006$ & 71 & $\mathrm{RF}$ & Brasil \\
\hline Soana (2011) & 2005 & 20 & $\mathrm{RSC}$ & Internacional \\
\hline Auamnoy \& Areepium (2011) & & 43 & $\mathrm{RF}$ & \\
\hline Oh, Chang, \& Martynov (2011) & 2006 & 118 & $\mathrm{RSC}$ & Corea \\
\hline Wang \& Qian (2011) & $2001-2006$ & 1465 & RSC & China \\
\hline $\begin{array}{l}\text { Wong, Ormiston, \& Tetlock } \\
\text { (2011) }\end{array}$ & $1996-2002$ & 61 & $\mathrm{RSC}$ & \\
\hline $\begin{array}{l}\text { Hamid, Akash, Asghar, \& } \\
\text { Ahmad (2011) }\end{array}$ & $2005-2006$ & 166 & $\mathrm{RF}$ & Pakistán \\
\hline Inoue \& Lee (2011) & $1992-2007$ & $74 / 183$ & $\mathrm{RF}$ & \\
\hline $\begin{array}{l}\text { Sahin, Basfirinci, \& Ozsalih } \\
\text { (2011) }\end{array}$ & 2007 & 165 & Ambas & Turquía \\
\hline
\end{tabular}


(Continuación).

\begin{tabular}{|c|c|c|c|c|}
\hline Artículo & Periodo estudiado & $\begin{array}{l}\text { Tamaño } \\
\text { muestral }\end{array}$ & $\begin{array}{l}\text { Variable } \\
\text { dependiente }\end{array}$ & Ámbito Espacial \\
\hline Usunier et al. (2011) & & $\begin{array}{l}93 / 38 / 96 / \\
23 / 52 / 190 \\
84 / 101 / 105 \\
99 / 45 / 110 / \\
106 / 65 / \\
42 / 556\end{array}$ & & $\begin{array}{l}\text { Alemania, } \\
\text { Australia, } \\
\text { Bahamas, } \\
\text { Brasil, China, } \\
\text { Dinamarca, } \\
\text { Francia, } \\
\text { Hong Kong, } \\
\text { Hungría, } \\
\text { India, } \\
\text { Jamaica, } \\
\text { Holanda, } \\
\text { Nueva } \\
\text { Zelanda, } \\
\text { Panamá, } \\
\text { Reino Unido } \\
\text { y EE.UU. }\end{array}$ \\
\hline $\begin{array}{l}\text { Guenster, Bauer, Derwall, \& } \\
\text { Koedijk (2011) }\end{array}$ & $1997-2004$ & $154-519$ & $\mathrm{RF}$ & EE.UU \\
\hline Melo (2012) & $2000-2005$ & 295 & $\mathrm{RSC}$ & EE.UU. \\
\hline $\begin{array}{l}\text { Godos-Díez, Fernández-Gago, \& } \\
\text { Cabeza-García(2012) }\end{array}$ & 2008 & 128 & $\mathrm{RSC}$ & España \\
\hline Purnomo \& Widianingsih (2012) & $2006-2010$ & 10 & $\mathrm{RF}$ & Indonesia \\
\hline Ahmad \& Ramayah (2012) & & 212 & $\mathrm{RF}$ & Malasia \\
\hline Dam \& Scholtens (2012) & 2005 & 691 & $\mathrm{RSC}$ & Europa \\
\hline Garay \& Font (2012) & 2009 & $302-307$ & & España \\
\hline Lanis \& Richardson (2012) & $2008-2009$ & 408 & & Australia \\
\hline Melo \& Garrido-Morgado (2012) & $2003-2007$ & 320 & $\mathrm{RSC}$ & EE.UU. \\
\hline Moroney, Windsor, \& Aw (2012) & 2003-2007 & 74 & $\mathrm{RSC}$ & Australia \\
\hline
\end{tabular}

